Argumentation et Analyse du Discours

\title{
Discours manifestant et contestation universitaire
} (2009)

Demonstration Discourse and University Contention in France (2009)

\section{Yana Grinshpun}

\section{OpenEdition}

1 Journals

Édition électronique

URL : http://journals.openedition.org/aad/1476

DOI : 10.4000/aad.1476

ISSN : $1565-8961$

Éditeur

Université de Tel-Aviv

\section{Référence électronique}

Yana Grinshpun, «Discours manifestant et contestation universitaire (2009) », Argumentation et Analyse du Discours [En ligne], 10 | 2013, mis en ligne le 10 avril 2013, consulté le 23 septembre 2019 URL : http://journals.openedition.org/aad/1476 ; DOI : 10.4000/aad.1476

Ce document a été généré automatiquement le 23 septembre 2019.

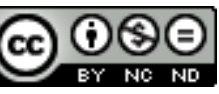

Argumentation \& analyse du discours est mis à disposition selon les termes de la licence Creative Commons Attribution - Pas d'Utilisation Commerciale - Pas de Modification 4.0 International. 


\title{
Discours manifestant et contestation universitaire (2009)
}

Demonstration Discourse and University Contention in France (2009)

\author{
Yana Grinshpun
}

1 Il existe en France une longue culture de contestation qui a constamment stimulé la créativité linguistique et discursive. Il suffit de songer au nombre impressionnant de mazarinades diffusées pendant la période de la Fronde $(1648-1653)^{1}$, aux placards et les journaux révolutionnaires à la fin $\mathrm{du} 18^{\mathrm{e}}$ siècle, à la production discursive massive lors de la Commune de Paris, aux publications anarchistes à partir du $19^{\mathrm{e}}$ siècle... Même s'ils ne sont pas aussi prolifiques, les mouvements sociaux, dès qu'ils prennent quelque ampleur, sont indissociables d'une production discursive spécifique. Il n'en va pas différemment au début du $21^{\mathrm{e}}$ siècle, et ceci d'autant plus qu'Internet lui offre de nouveaux espaces. Dans cet article, nous sommes particulièrement intéressée au mouvement contestataire universitaire qu'a connu la France en 2009, en fixant notre attention sur les énoncés montrés au cours des manifestations.

2 "Le 2 février l'université s'arrête » : ce mot d'ordre est lancé en réponse aux projets de réformes proposées par V. Pecresse et X. Darcos et aux vœux que N. Sarkozy a adressés aux enseignants chercheurs le 22 janvier 2009. Ces réformes découlent de la convergence de deux processus connus sous le nom de déclaration de Bologne et de stratégie de Lisbonne qui ont conduit à une restructuration de l'enseignement supérieur européen, salué par certains comme une nécessaire modernisation, dénoncé par d'autres comme une marchandisation croissante du savoir et une subordination de la recherche aux impératifs des grands groupes économiques privés. A cela s'ajoute une réforme profonde de la formation des enseignants qui supprime l'année de formation en alternance pour les étudiants des Instituts de Formation des Maîtres reçus aux concours de professeurs. Cette dernière réforme, mal préparée, est très particulièrement critiquée dans les facultés de lettres.

3 Les mouvements universitaires contestataires commencent en France en 2007 avec l'entrée en vigueur de la loi relative aux libertés et responsabilités des universités (LRU). Cette loi, promulguée en 2007 est également appelée la Loi Pécresse, du nom de 
la Ministre de la recherche. En 2009, le gouvernement essaie de faire entrer en vigueur les décrets d'application de cette loi, qui entend réformer le statut des enseignantschercheurs et la gestion des universités, en particulier en ce qui concerne leur budget, et institue la «masterisation» des concours des professeurs de l'enseignement secondaire. Ces réformes se détachent sur un fond de malaise, lié à une profonde remise en cause du statut des enseignants-chercheurs de l'université ; les critiques se portent, en particulier dans les facultés de lettres, sur l'AERES (Agence d'Evaluation de la Recherche et de l'Enseignement Supérieur) accusée d'évaluer la recherche en fonction de critères purement quantitatifs.

4 Au cours du deuxième semestre 2009 de nombreux mots d'ordre de grève générale sont lancés dans l'université pour s'opposer aux mesures gouvernementales. Mais dans la pratique ces grèves paralysent surtout les facultés de lettres et de sciences humaines et sociales, les plus sensibles aux conséquences des réformes.

5 La contestation a des résultats mitigés. Le gouvernement recule sur certains points de la réforme, en particulier sur le statut des enseignants-chercheurs et leur évaluation, mais le cadre d'ensemble de la réforme est maintenu. Les participants ont le sentiment d'un échec, dû selon eux au manque de soutien de l'ensemble des personnels universitaires et des syndicats de l'enseignement secondaire, pourtant concernés par la réforme de la formation des professeurs.

Dix manifestations au deuxième semestre 2009 (la première date du 10 février 2009 et la dernière du 5 mai 2009) qui associent enseignants et étudiants sont organisées dans la région parisienne. Participante active du mouvement contestataire, j'ai suivi toutes ces manifestations. Mon attention de linguiste et d'analyste du discours a été tout naturellement attirée par les caractéristiques de ce qu'on pourrait appeler le "discours manifestant». La manifestation (politique, syndicale...) est en effet une situation de communication très particulière qu'on peut appréhender à la fois comme construction d'une communauté de conviction transitoire (elle n'existe en effet que le temps de la manifestation), et comme espace de production et de «monstration » d'énoncés oraux et écrits. Une particularité de ce type de manifestation est qu'elles sont hétérogènes : elles intègrent à la fois des groupes, en particulier d'ordre syndical, et des individus qui ne sont associés à aucun appareil.

7 Traditionnellement, on associe manifestation et slogan. Il existe un nombre important de travaux qui abordent ce phénomène du point de vue linguistique ou rhétorique. Beaucoup de chercheurs se sont intéressés à l'argumentation : Adam (1997), EveraertDesmedt (1982), Delbecque et Leuven (1990), Herrero Cecilia (1995); d'autres se sont penchés sur les aspects plus linguistiques du slogan: Bachala, Bentolila, Carvalho (1977), Grunig (1990), Garric (1996), Herrero Cecilia (1997), etc. Nous ne pouvons résumer tous ces travaux dans le cadre de notre article, le lecteur trouvera les références dans la bibliographie. Il faut néanmoins reconnaître que dans la littérature spécialisée, en tout cas en français, il n'existe guère de recherches sur le slogan qui s'appuient sur des corpus attestés recueillis lors de mouvements contestataires d'envergure. Quant à l'ouvrage de référence La manifestation dirigé par Pierre Favre (1990), il privilégie les points de vue historique et sociologique. Les caractéristiques de la parole manifestante n'y sont pas vraiment abordées. Les chercheurs qui ont contribué à ce volume se sont surtout intéressés aux phénomènes sociaux en amont et en aval de l'événement «manifestation » qu'ils analysent à la fois comme une action symbolique et comme une stratégie. 
Nous allons nous baser ici sur un corpus d'énoncés que nous avons constitué pendant les manifestations universitaires du printemps 2009. L'objectif principal de cet article est de mettre en évidence, sur un aspect particulier (les énoncés portés par les manifestants), la spécificité de ce mouvement de contestation. A la différence de la plupart des recherches sur les slogans, nous ne nous contentons pas d'analyser les procédés qui permettent de les construire, nous les abordons comme relevant d'une pratique inscrite dans une certaine conjoncture historique, sociale et politique. De ce point de vue, en effet, l'analyse énonciative seule ne sera pas suffisante: il faut en particulier prendre en compte le statut et le positionnement des acteurs. Nous donnons ainsi la priorité à la dimension sociale des énoncés, appréhendés dans le cadre communicationnel de la manifestation. Nous travaillerons donc dans une perspective d'analyse du discours, qui vise à articuler fonctionnements linguistiques et situations de communication à travers les dispositifs d'énonciation.

\section{Slogans et énoncés manifestants}

9 Pour la clarté du propos, il nous faut définir quelques termes qui sont essentiels pour cet article. Nous appelons ici «discours manifestant» l'ensemble de la production sémiotique (verbale ou iconique) d'une manifestation ou d'une série de manifestations qui ont un même objectif. Nous entendrons par "énoncés manifestants » l'ensemble des énoncés verbaux scandés par les manifestants ou inscrits sur les banderoles ou les affichettes. Les slogans ne sont qu'un sous-ensemble de ces énoncés manifestants. En effet le slogan prototypique d'une manifestation, tel qu'il est habituellement décrit dans les travaux des spécialistes, est une formule concise dont le signifié et le signifiant ont une forme prégnante, destinée à être scandée oralement et de manière collective. Or beaucoup d'énoncés manifestants ne présentent pas ces caractéristiques; ils ne sont contestataires que de manière indexicale, parce qu'ils s'inscrivent dans le contexte de la manifestation dont ils participent.

\subsection{L'approche classique du slogan}

10 Sur le slogan politique il est de rigueur de mentionner les travaux du philosophe et spécialiste de la rhétorique, Olivier Reboul, auteur d'un ouvrage intitulé précisément $L e$ slogan (1975). Il le définit de la manière suivante :

une formule concise et frappante, facilement repérable, polémique et le plus souvent anonyme, destinée à faire agir les masses tant par son style que par l'élément d'autojustification, passionnelle ou rationnelle, qu'elle comporte : comme le pouvoir d'incitation du slogan excède toujours son sens explicite, le terme est plus ou moins péjoratif (1975:42).

11 Sa réflexion sur les propriétés linguistiques des slogans reste néanmoins allusive. Elle porte pour l'essentiel sur sa « concision ». Ce type de formatage est pour lui associé à certains effets cognitifs. "Il s'agit d'un pouvoir spécifique. Pour le comprendre, revenons à l'un des termes de la définition du slogan : sa concision. Elle lui est essentielle. Allonger un slogan n'est pas le renforcer mais l'affaiblir, parfois le détruire [...]» (48). Reboul ne raisonne pas en termes de genre de discours, mais en termes rhétoriques, insistant sur l'impact qui est produit par ce type d'énoncés et sur les stratégies argumentatives qu'ils impliquent, en particulier le travail sur les lieux communs. Il souligne également leur lien avec l'inconscient, surtout lorsqu'il aborde la 
dimension ludique de nombreux slogans. Sur ce point, il fait référence aux travaux de Freud sur le mot d'esprit et sur les rêves.

Freud affirme d'autre part que le mot d'esprit se distingue du rêve par le fait qu'il déjoue une censure non pas endormie mais vigilante. Le slogan semble quant à lui bien plus proche du rêve et de la régression qui lui est propre. Il assure presque toujours le triomphe de l'imagination sur la raison, du principe de plaisir sur celui de la réalité. Aussi peut-on comprendre le pouvoir des figures qu'il emploie, notamment des plus constantes, la métaphore et l'hyperbole. La première est en effet, un des grands procédés du rêve pour déjouer la censure: elle permet la dramatisation ou régression du concept de l'image, de la pensée adulte à la pensée enfantine : le slogan joue sur le procédé en changeant la lame de rasoir en grande amoureuse. Quant à l'hyperbole, elle plaît comme retour à la mentalité enfantine, qui ne distingue pas le comparatif du superlatif. [...] Certains slogans s'imposent par leur ton catégorique : n'est-ce pas qu'ils font retrouver en nous la voix impérieuse et rassurante des parents? $(1975: 86)$

Quelque intéressante que soit cette analyse du plaisir régressif associé au slogan (on en trouve de nombreuses illustrations dans notre corpus, par exemple: «Pécresse, serre tes fesse, on arrive à toute vitesse »), elle laisse de côté de nombreuses questions quant aux conditions de production des slogans ainsi qu'aux procédés linguistiques qu'ils mobilisent. Cela s'explique aussi par l'objectif de l'auteur qui ne s'appuie pas sur un corpus, et qui mêle des considérations qui portent à la fois sur le discours politique et sur le discours publicitaire.

Une des raisons majeures qui rendent l'approche de Reboul peu opératoire pour l'analyse des manifestations universitaires qui nous intéressent est qu'il ne prend en compte que les slogans "prototypiques" politiques, ceux qui sont destinés à être scandés collectivement et qui s'adressent à «l'homme des masses » (92). Il n'en va pas différemment dans l'étude de Denton (1980), qui adopte lui aussi une perspective rhétorique et propose une typologie. Il souligne les diverses fonctions du slogan politique, qui simplifie les idées complexes, exprime l'idéologie de groupes, provoque l'identification, structure et active la confrontation et ouvre des perspectives d'avenir. Or, dans notre corpus, on est obligé de distinguer entre deux catégories d'énoncés manifestants, la seconde ne correspondant pas véritablement à des slogans :

1. Les slogans "prototypiques", ceux qu'analyse Reboul : c'est le cas par exemple de "Universidad unida jamás será vencida », captation d'un slogan latino-américain bien connu. Ce type de slogan passe en règle générale par des schémas préétablis qui fonctionnent comme des matrices : « X, non non non, $Y$ oui oui oui », « $\mathrm{X}$ avec nous », « non à X ", etc.

2. Les slogans qu'on pourrait dire "scripturaires » : ils sont écrits sur des banderoles, mais ne sont pas scandés. Ils suivent eux aussi des schémas routiniers qui permettent de les reconnaître comme tels. En particulier ceux du type « $X$ en + Nom » :

$\mathrm{X}$ en colère

$\mathrm{X}$ en danger

$\mathrm{X}$ en lutte...

15 En outre comme on va le voir, ces deux catégories d'énoncés manifestants ne représentent qu'une partie du corpus. Les énoncés sur les affichettes portées individuellement leur échappent. 


\section{2. L'hyperénonciateur du slogan}

Dans ses travaux récents D. Maingueneau (2012) a abordé les slogans comme «particitations », comme sous-catégorie des « aphorisations ». Pour lui, l'« énonciation aphorisante » est un type d'énonciation spécifique : entre un énoncé « aphorisé » et un texte il n'y a pas une différence de taille, mais d'ordre. Les slogans, comme les proverbes, sont des énoncés détachés par nature, à la différence des aphorisations qui sont détachées de textes (ainsi les citations célèbres : "I have a dream » "La religion est l'opium du peuple »...). Maingueneau caractérise la «particitation » à l'aide des critères suivants :

- L'énoncé cité est mémorisable et autonome, par nature ou par détachement d'un texte.

- Il prétend être reconnu comme citation par les destinataires, sans que le locuteur citant indique la source, et sans même qu'il précise qu'il effectue une citation à l'aide d'un verbe de dire introducteur, d'une incise. Le caractère de citation est seulement marqué par un décalage interne à l'énonciation, qui peut être signalé sur le plan graphique, phonétique, paralinguistique.

- L'énoncé cité est présenté dans son signifiant dans une logique du discours direct, mais poussée à l'extrême: il ne suffit pas de simuler une énonciation autre, comme c'est souvent le cas au discours direct, il faut restituer le signifiant même. Cette restitution peut néanmoins s'accommoder de variation, comme on l'a souvent montré pour des formes pourtant communément considérées comme figées, les proverbes. La nécessité de rendre le signifiant est évidemment liée au fait qu'il n'y a pas d'indication de la source de la parole rapportée.

- Il appartient à un thésaurus verbal aux contours flous, indissociable d'une communauté qui, précisément, se définit de manière privilégiée par le partage d'un tel thésaurus. Par son énonciation, le locuteur citant présuppose pragmatiquement que lui-même et son allocutaire sont membres de cette communauté qu'ils sont pris dans une relation de type spéculaire : le locuteur cite ce qui pourrait être dit par l'allocutaire et, au-delà, par tout membre de la communauté qui agit de manière pleinement conforme à cette appartenance.

- Ce thésaurus et la communauté correspondante implique un hyperénonciateur, qui leur donne leur unité et dont l'autorité garantit moins la vérité de l'énoncé-au sens étroit d'une adéquation à un état de choses du monde - mais plutôt sa validité, son adéquation aux valeurs, aux fondements d'une collectivité. (Maingueneau, $2012: 60$ )

Les slogans politiques sont rangés parmi les "particitations militantes », qui relèvent elles-mêmes des «particitations de groupe " (2004: 119). Énonciation collective, la particitation de groupe "renforce la cohésion d'une collectivité en l'opposant à un extérieur menaçant ». Ici le collectif doit être analysé sur trois niveaux distincts :

1. Les locuteurs empiriques composant le groupe des manifestants

2. L'acteur collectif dont ils participent (syndicat, amicale, profession...)

3. L'hyperénonciateur qui fonde l'ensemble des points de vue qui soutiennent les énoncés du discours manifestant : « la Gauche », « la Nation », « le Parti » etc.

Dans le cas de la manifestation, la collectivité n'est pas une institution préétablie mais une communauté transitoire qui n'existe que par cette manifestation même. En dépit de son caractère transitoire, cette communauté implique toutefois un hyperénonciateur qui « doit exister au-delà de ce rassemblement fugace, assurer une 
continuité imaginaire d'un rassemblement à l'autre" (2004: 120). Maingueneau souligne toutefois la tension entre une tendance à la fragmentation (la manifestation se divise en composants qui ont leurs slogans et leur hyperénonciateur spécifiques) et une tendance à l'unité (un seul hyperénonciateur pour tous). Dans notre corpus nous allons effectivement voir qu'il en est ainsi.

19 L'approche de Maingueneau présente l'avantage de mettre au centre la collectivité associée au slogan et le dispositif énonciatif que cela implique, en particulier l'existence d'un "hyperénonciateur». Mais, comme Reboul, il limite son propos aux slogans classiques; il n'appréhende pas les énoncés manifestants dans leur diversité, comme le montre le corpus sur lequel nous avons travaillé.

\subsection{Le corpus}

Ce corpus est constitué de 148 énoncés manifestants recueillis dans les cortèges parisiens $^{2}$ des manifestations étudiantes entre février et juin 2009. Malgré le souci de " couvrir » tous ces cortèges (une dizaine), nous ne pouvons en aucun cas prétendre à l'exhaustivité. Etant donné la nature de ces événements, il paraît de toute façon difficile de viser l'exhaustivité. Le choix que nous avons fait de photographier systématiquement les énoncés à notre portée au cœur des manifestations nous semble suffisant pour que l'on puisse parler de corpus représentatif. En tout cas, il est suffisant pour le type d'analyse que nous menons ici.

Comme ce corpus est basé sur des photographies, il présente l'avantage de prendre en compte des phénomènes qui vont au-delà du domaine strictement linguistique. C'est ainsi que nous avons opéré une distinction entre deux sous-corpus majeurs : celui des banderoles collectives et celui des affichettes individuelles portées par les participants. Un analyste du discours ne peut en effet ignorer l'importance des supports dans le processus de communication: "le médium n`est pas un simple moyen de transport pour le discours, il contraint ses contenus et commande les usages qu'on eut en faire. [...] une transformation importante du medium modifie l'ensemble d'un genre de discours. " (Maingueneau 1998 : 57). En procédant ainsi, nous donnons de l'importance à un trait qui est largement ignoré par les typologies usuelles des slogans, fondées sur les critères linguistiques et rhétoriques. Nous sommes ainsi amenée à appréhender ce corpus à travers une grille en apparence secondaire mais qui, nous semble-t-il, révèle des aspects intéressants de ce cycle de manifestations. Les énoncés du corpus peuvent être divisés en deux grandes catégories :

- les énoncés purement verbaux

- les énoncés icono-verbaux, où le verbal est inséparable du visuel.

On trouve peu de slogans purement iconiques, à quelques exceptions près où il s’agit d'effigies de protagonistes des réformes : Sarkozy et Pécresse. Cela est peut-être lié au fait qu'il s'agit majoritairement de manifestants issus des facultés de lettres; le maniement de la parole est leur « arme » privilégiée.

Comme nous l'avons déjà dit, les énoncés manifestants sont loin de se présenter tous comme des slogans, en donnant à ce terme un contenu assez large, de manière à recouvrir à la fois les slogans prototypiques (scandés) et les slogans que nous avons dits "scripturaires ». Ils obéissent à un schéma préformaté. Trois d'entre eux se détachent nettement: «Non à... », « X = Y », « $X$ en danger ». L`utilisation de ces schémas a une triple fonction : 
- De manière réflexive, elle permet au slogan de se montrer comme slogan, ce qui est nécessaire pour réussir l'activité communicationnelle.

- Elle permet aux manifestants de montrer leurs capacités de militant, à travers leur compétence discursive : ils savent produire des énoncés conformes aux normes de la situation de communication et par là même se convertir en manifestants légitimes.

- Elle leur permet de s'inscrire dans une filiation imaginaire, de montrer leur appartenance à une tradition de contestation. On retrouve là l'hyperénonciateur et le thésaurus évoqués par D. Maingueneau.

\section{Le support}

\subsection{Les énoncés manifestants sur les banderoles collectives}

Nous appelons "banderoles collectives » un long morceau de tissu ou de papier porté par un ensemble de personnes qui appartiennent au même groupe (CNRS, fac, département, syndicat...). Ces énoncés sont au nombre de 24 , ce qui ne représente qu'environ $16 \%$ du corpus. Sur ces banderoles on peut trouver seulement le nom du collectif (« Université Paris 7 UFR LCAO », «Intersyndicale Université Paris III »...), ou seulement des slogans ("Non aux suppressions d’emplois! Défendons l'Université !", « Non à l'Université bling-bling! »), ou la combinaison des deux (« CNRS en lutte»).

\subsection{Affichettes individuelles}

Il s'agit d'affichettes portées par une personne, souvent attachées sur son corps: affichettes attachées à la tête en forme de chapeau, aux épaules, au dos, à la poitrine et même aux fesses. D'un point de vue anthropologique, à l'instar de ce qui se passe avec les tatouages l’endroit du corps auquel est attachée l`affiche a de l'importance. La question plus générale qui est ainsi posée est celle de l'«incorporation», de l'appropriation de l'énoncé par le corps des manifestants. Cette incorporation s'opère à un premier niveau, collectif (le manifestant est un marcheur qui montre son corps à tous), et à un second niveau, individuel (les participants peuvent adresser un message implicite par la manière dont ils mettent en relation leurs corps et leurs énoncés : banderoles au-dessus des têtes ou affichettes apposées sur telle ou telle partie du corps). On peut penser que ce phénomène relève de la « corpographèse " (Paveau 2009; Paveau et Zoberman éds 2009), mais au sens large. Car à la différence de "l'écriture cutanée » (Paveau) du tatouage, porterait sur le corps habillé et serait transitoire. Nous ne pouvons malheureusement pas développer ce point ici.

Ces énoncés, au nombre de 113, représentent $71 \%$ des énoncés manifestants de notre corpus. Exemples :

Il faut dépecresser le mammouth

La rage!!!! (avec une photo de Pasteur)

Doctorat ha ha ha

Les énoncés de cette seconde catégorie ne sont pas homogènes. Certains pourraient figurer sur des banderoles collectives. Par exemple :

Paris 3 contre le déclin de l'Université

D'autres non :

L'autonomie tuera notre liberté

C'est vous les réacs c'est pas nous 
Seulement dix $(8,8 \%)$ d'entre eux se présentent néanmoins comme des slogans prototypiques, tels que :

Non à la star' fac

Géographes en colère

Dans l'immense majorité des cas (97\%), ces affichettes ne mentionnent pas d'auteur collectif facilement repérable (Paris III, CNRS...). Ce type d'énoncés doit en effet être rapporté non à un groupe particulier qui servirait de médiateur et dont le locuteur serait un représentant, mais directement à l'hyperénonciateur supposé qui confère son unité et sa raison d'être à la manifestation.

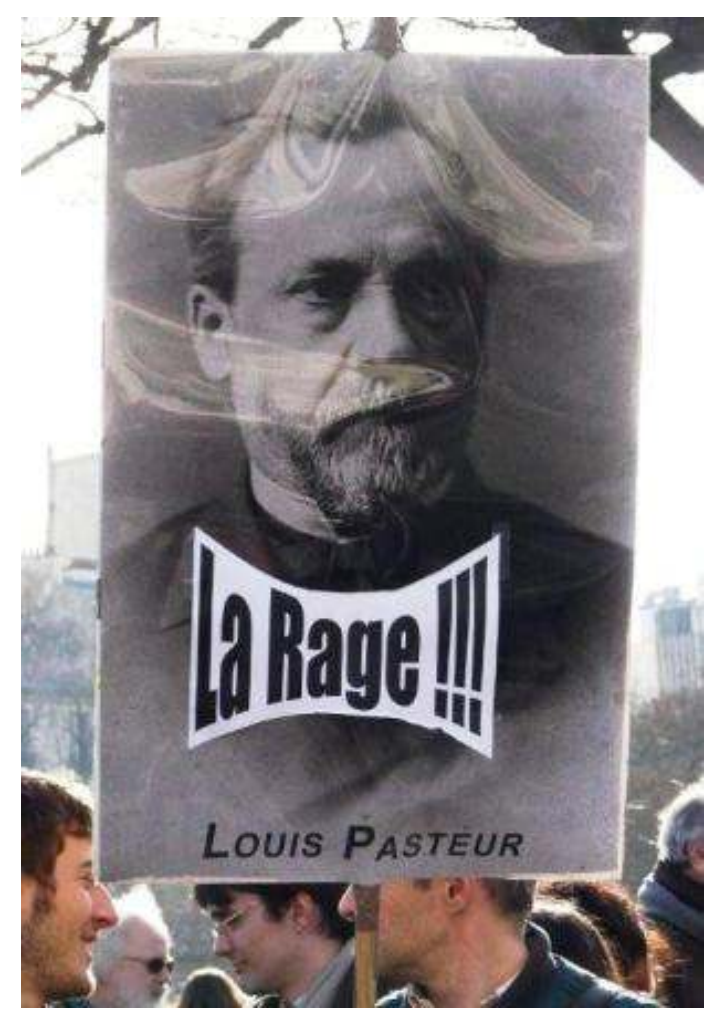

« La rage !!! »

Photo Yana Grinshpun

\section{3. Les affichettes portées par deux ou trois personnes}

29 Ces énoncés sont au nombre de 11 , soit $6,9 \%$ du corpus. En voici deux exemples :

Esprit critique sens unique

Et là tu nous vois? (Au-dessus du slogan on voit une caricature de Sarkozy)

Comme on peut s'y attendre, leur inscription dans le discours manifestant se fait de la même manière que les affichettes individuelles. Seulement trois sur onze se présentent comme des slogans. Par exemple:

Non à la suppression des maternelles

\section{Les deux communautés}

30 Le problème est de savoir quelle est la communauté correspondante, car il peut y en avoir plusieurs ; ce qui semble bien être le cas ici. En effet, ces manifestations semblent caractérisées par une tension entre un cadre syndical classique (« Non aux suppressions 
d'emplois. Défendons l'Université !») de "défense du service public» et un grand nombre de productions individuelles qui impliquent l'appartenance à une communauté imaginaire 'humaniste' - au sens des «humanités » - au service d'une défense de «la culture ». A l'évidence c'est cette dernière qui est dominante. On saisit sur le vif ici comment un groupe met son capital symbolique au service de luttes qui ont précisément pour visée de défendre les conditions qui favorisent la préservation de ce capital. C'est à travers les catégories de ce capital qu'il effectue une polarisation entre ses propres valeurs et les valeurs contraires prêtées à l'adversaire qu'il construit dans son discours. La mobilisation de ce capital symbolique se manifeste à deux niveaux complémentaires: 1) par un usage sophistiqué de la parole, 2) par le recours multiforme et réitéré à une topique qui oppose "culture » et "argent ", à travers des énoncés tels que

La culture n'est pas une marchandise

Le cerveau n`a pas de prix et le savoir est à tous (cet énoncé apparaît avec un cerveau sur lequel on voit un code-barre).

Manet Monet Money

Mon cerveau n`est pas à vendre

Non à la star'fac

31 Tous ces exemples proviennent précisément des affichettes individuelles. L'énoncé "Manet Monet Money» joue sur le signifiant du nom de deux grands peintres emblématiques d'une culture raffinée, censés dégradés en "money», l'usage de l'anglais connotant ici l'économie capitaliste.

32 Cette topique qui oppose culture et argent entre dans un paradigme de lieux communs rhétoriques qui se mêlent et s'appuient les unes les autres :

Qualité vs Quantité

Esprit vs Matière

Savoir vs Ignorance

Etre vs Paraître..

Ce paradigme d'oppositions est sous-jacent à la plupart des énoncés manifestants qui visent les protagonistes de nouvelles réformes et les valeurs mercantiles qu'ils incarnent aux yeux de la communauté des 'humanistes'. Les personnages les plus visés sont naturellement Sarkozy, le président de la République, et ses ministres, Valérie Pécresse, ministre de la Recherche et de l'Enseignement supérieur, et Xavier Darcos, ministre de l'Education nationale ${ }^{3}$ :

Illiteratus rex quasi asinus coronatus est

Pécresse nuit à la culture

Darcos nuit a l'école républicaine

Ignorance au pouvoir = perte de Savoir

Ce type d'énoncés légitime l'identité imaginaire des acteurs-porteurs des slogans en justifiant en même temps leur positionnement dans une lutte contre le «matérialisme» du pouvoir. Il active l'ethos de la communauté des savants et des lettrés qui sont censés ériger le savoir et la culture en valeurs suprêmes, constamment menacées par le pouvoir de l'argent. Cet ethos lettré s'inscrit bien évidemment dans une tradition qui fournit des "modèles culturels prégnants", comme le souligne R. Amossy :

les rôles qu'endosse délibérément le locuteur dans le scénario de son choix font partie d'un arsenal préexistant. Ils répondent à des modèles culturels prégnants (le pater familias, l'homme du peuple qui dit la vérité nue) en se référant aux représentations collectives du groupe. C'est dire que l'image de soi est doublement 
déterminée, à la fois par les règles de l'institution discursive et par un imaginaire social. Dans la mesure où elle s'élabore dans des cadres contraignants en fonction de modèles culturels entérinés, elle témoigne de la force de l'institution et de l'idéologie ambiante. [...] On fait l'hypothèse que l'ethos, comme l'énonciation, le dialogisme ou l'argumentativité, est une dimension constitutive du discours. En tant que tel, il est en relation dynamique avec les autres dimensions constitutives: il est ancré dans l'énonciation, il est foncièrement dialogique et nécessairement doté d'une dimension, sinon d'une visée argumentative (Amossy $2010: 38,42$ ).

Par cet ethos, les énonciateurs qui appartiennent à la même communauté mettent aussi en scène l`anti-ethos ${ }^{4}$ de ceux qui n`adhèrent pas à leurs valeurs.

L'interprétation de ces énoncés relève de deux régimes interprétatifs à la fois. D'une part, elle relève d'un régime "d'actualité » (Maingueneau 2012 : 108), pour ceux qui disposent du savoir encyclopédique nécessaire, ont accès à un vaste interdiscours médiatique (les propos oraux de membres du gouvernement, les décrets publiés dans la presse, les communiqués des ministres et des présidents de l'Université...). Sur ce point, le discours manifestant permet de souder la communauté : les énoncés sont tournés davantage vers l'intérieur que vers l'extérieur. L'interprétation relève d'autre part d'un régime " mémoriel » : les énoncés qui opposent le savoir à l’argent mobilisent un répertoire connu du public cultivé. L`ensemble des énoncés qui portent par exemple sur le roman La Princesse de Clèves ${ }^{5}$ ne peuvent être compris que par un public restreint, qui par cette connivence renforce sa cohésion.

Mais la connaissance de l'actualité et de l'histoire littéraire ne suffisent pas. Hors contexte, très peu de gens, même parmi les enseignants du secondaire, pourraient interpréter les énoncés manifestants tels que ceux-ci :
La princesse crève
Libérez la princesse de Clèves
Le duc de Nemours vous rend « vos preuves d'amour »

On se rappelle que Nicolas Sarkozy s'en est pris à la mise au programme du roman de Mme de La Fayette dans certains concours administratifs. Ses attaques se sont renouvelées dans trois interventions, entre 2006 et 2009. En 2009, les propos du président qu'il a tenus sur La princesse de Clèves seront perçus par la communauté universitaire comme emblématiques de la politique de Valérie Pécresse et Xavier Darcos.

39 Pour interpréter de tels énoncés il faut également prendre en compte l'activité manifestante même où ils apparaissent, leur dimension indicielle. Ici la princesse fonctionne comme désignateur indirect des manifestants 'humanistes' et, au-delà, du monde qui est le leur et dont participe précisément la connaissance du roman de Mme de Lafayette.

Prenons quelques énoncés significatifs à titre d'exemples. Si l'on excepte les énoncés formulaires, il est très difficile de distribuer les énoncés manifestants dans une série de catégories étanches. Ces créations la plupart du temps ludiques déjouent les frontières établies et associent divers procédés. C'est pourquoi les chercheurs qui s'intéressent à ce type d'énoncés préfèrent recenser ces procédés et analyser quelques exemples. Dans le contexte où la communauté universitaire " conteste ", le discours des manifestations est constamment traversé par du déjà-dit. Il s'agit donc de voir comment est agencée la pluralité des voix au sein des mêmes énoncés. Nous nous appuierons à la fois sur les théories de la polyphonie linguistique et du dialogisme (Ducrot, 1984 ; Bres, 2005) et sur la problématique de la particitation. 
41 Étant donné que les manifestations que nous étudions ont été dominées par des étudiants et des enseignants issus des facultés de lettres, il n`est pas surprenant que les énoncés attestent d'une grande créativité, qui mobilise des ressources très diverses: des jeux sur la polyphonie, abondamment décrits dans la littérature spécialisée, à des jeux sur la morphologie, en passant par l'intertextualité et le recours à des langues étrangères. Ici discours politique et discours littéraire sont étroitement liés, impliquant une certaine lecture 'humaniste' du réel.

\section{Les langues étrangères}

42 Tout au long des manifestations, nous avons vu nombre de slogans en langues étrangères. Parmi les langues vivantes, prédominent l’anglais et l’espagnol, parmi les langues mortes, c’est essentiellement le latin qui est attesté.

\subsection{L'anglais et l'espagnol}

Dans le cas des slogans qui font appel à l'anglais, il peut s’agir de captation d'aphorisations célèbres, en particulier « Yes we can » de Barak Obama énoncé en 2008 à Nashua (New Hampshire) : « Sauver l'université, yes we can, détruire l'université, no he can't ». Rappelons que la captation d'un texte implique une imitation qui est censée aller dans le même sens que l'imité. Quand on insère dans l'énoncé la citation en anglais d'Obama, on entend signifier qu'on participe de la même lutte que lui, on attribue implicitement le même hyperénonciateur aux deux énonciations. Cette captation prend tout son sens par le fait que l'anglais (cf. "Manet Monet money ») est précisément la langue de ceux qui au nom des valeurs du néo-libéralisme promeuvent la réforme de l'université française.

On retrouve une autre forme de captation dans le slogan en espagnol «La Universiad unida jamás será vencida ", qui capte l'aphorisation détachée d'une chanson écrite en 1973 au Chili par Sergio Ortega : «El pueblo unido jamás será vencido ». Cette aphorisation est devenue un des slogans emblématiques des manifestations de gauche depuis les années 1970; la substitution de «universidad» à «pueblo» permet d'assurer la continuité avec un même hyperénonciateur. Et ceci d'autant plus qu'en dernière instance, ce sont les U.S.A. qui inspirent les adversaires de ceux qui profèrent ce slogan : les promoteurs du coup d'Etat au Chili, d'une part, ceux de la réforme qui vise à une « marchandisation » des universités, d'autre part.

La célèbre aphorisation d'Obama est également mise au service d'une autre forme de captation, mais indirecte : « No you can’t détruire notre école ». Cet énoncé, négatif de l'autre, est présenté comme un énoncé potentiel d'Obama qui est assumé par les manifestants. La neutralisation en anglais de l'opposition française entre "tu » et «vous » fait que ce slogan peut viser aussi bien les adversaires de la communauté manifestante que le seul Sarkozy, à qui les 'humanistes' attribuent l'ethos d'un inculte, qui plus est ennemi de la culture.

\subsection{Le latin}

Considérons à présent cette banderole où Sarkozy est recatégorisé en âne :

Rex illiteratus quasi asinus coronatus est 
Il s’agit ici d’une affichette portée par plusieurs étudiants issus de la Sorbonne (Université Paris IV). Cet animal fonctionne comme une sorte d'emblème qui permet de fixer l'un des composants essentiels de l'ethos sarkozyen tel qu'il circule dans les médias. L'emploi du latin a pour effet d'inverser les rapports de force : à l'intérieur de la parole sarkozyenne la culture 'humaniste' est disqualifiée, mais à l'intérieur de l'univers 'humaniste', incarné dans l'usage du latin, le Président se transforme en âne. Le spectateur s'il a une grande culture classique, rapportera cet énoncé à sa source, l'encyclopédie morale Politicratus de Jean Salisbury (1156), qui participe du Thésauraus de l'hyperénonciateur 'humaniste'.

En règle générale, dans les situations de ce type, la supériorité du plaisantin suppose la création d'un lien de solidarité contre le tiers exclu (celui qui ne comprend pas le code) qui renforce la cohésion de la communauté. Manier avec dextérité les langues classiques et les références littéraires ou philosophiques est une manière de montrer son identité d' 'humaniste' cultivé tout en disqualifiant l'autre ignorant. L'énonciation contestatrice va de pair avec un mouvement d'autolégitimation. Il en va de même dans un énoncé tel que "Universitas delenda est », qui capte l'aphorisation célèbre " Carthago delenda est » de Caton l'Ancien lors des débats au sénat romain sur le sort de Carthage. Cet énoncé manifestant n'est pas pris en charge par ses porteurs, mais son point de vue est attribué aux ministres initiateurs des réformes. La polyphonie joue donc sur trois plans d'énonciation: celui de Caton, celui des promoteurs des réformes et celui des porteurs de l'affichette. La subtilité de ce dispositif tient au fait que, précisément, les promoteurs de la réforme sont les adversaires de la culture qui véhicule la phrase de Caton et la rend encore intelligible pour une communauté de privilégiés.

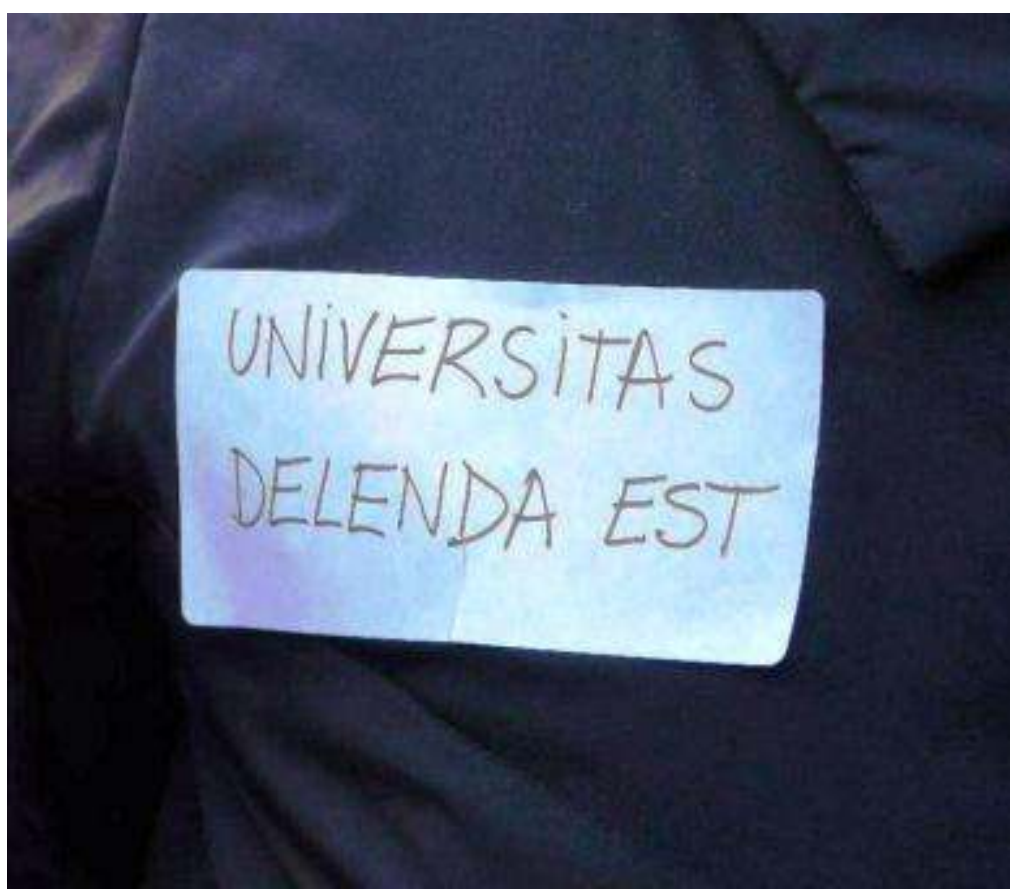

«Universitas delenda est »

Photo Yana Grinshpun

On le voit, il s'agit toujours de l'imitation ludique des textes ou de particitations extraits d'un Thésaurus 'humaniste'. Le renvoi à cet intertexte permet à la fois de montrer un ethos de locuteur lettré et de disqualifier l'adversaire dont le propre est 
l'anti-ethos (ignare, inculte, bling-bling, matérialiste, etc.) On le voit dans cette affichette qui joue sur la subversion d'une référence donnée sur le mode universitaire :

Sarkozy, N. \& Pécresse, V. (2009) Comment tuer l'université et la recherche en une loi et quelques décret? Revue d'inculture appliquée. Vol 3. (2) 23-45 impact factor.

\section{La princesse de Clèves}

L'affaire de La princesse de Clèves en particulier ne manque pas de stimuler la créativité des manifestants : à travers des pièces de théâtre, des cours donnés sur les places publiques, de nombreux clips diffusés sur You Tube et Daily Motion, des chansons, des caricatures, des marionnettes s'est imposé l'ethos d'un Sarkozy ennemi de la culture 'humaniste'. Cet ethos contamine ses ministres. Un slogan de la manifestation du 14 février 2009 (par ailleurs, jour de la Saint-Valentin) a pris pour cible le discours de la Ministre de l'Enseignement supérieur de l'époque, Valérie Pécresse: "Le duc de Nemours vous rend bien "vos preuves d'amour" ". C'est un cas de double subversion. V. Pécresse, dans un de ses discours sur l'enseignement supérieur avait cité un énoncé proverbial «il n'ya pas d'amour sans preuves d'amour. Et ces preuves nous [le gouvernement] nous vous les donnons tous les jours $»^{6}$. Cet énoncé a été tout de suite exploité par les adversaires de la réforme. Dans les autres énoncés manifestants qui mentionnent la princesse de Clèves, la communauté universitaire est en règle générale identifiée à la princesse, érigée en icône de la culture classique et de la résistance du mouvement universitaire. En revanche, dans le slogan que nous venons de citer, c'est au duc de Nemours, convoité par toutes les dames de la cour, que s'identifient les 'humanistes', en attribuant à Valérie Pécresse le rôle ridicule d'une amoureuse éconduite.

De manière plus large, l'affaire de la Princesse de Clèves peut être analysée en termes de construction de mémoire collective pour le groupe contestataire. Cette affaire devient, dans la parole protestataire, un lieu de mémoire partagée, que les énoncés manifestants ne cessent de réactiver et d'enrichir. Mais cela ne peut se faire qu'en accordant une place prépondérante à l'auditoire interne de la manifestation. L'auditoire externe, celui des non-manifestants, est d'ailleurs relégué à l'arrière-plan par le seul fait que les affichettes sont difficilement lisibles au-delà de l'environnement immédiat de leurs porteurs, à la différence des banderoles.

\section{Un cas particulier de polyphonie : les détournements littéraires}

51 Supposons qu'au cours d'une discussion entre gens cultivés, quelqu'un énonce avec une intonation spécifique une phrase de Corneille ou de Victor Hugo, avec la prétention qu'elle soit reconnue par ses allocutaires. En produisant une telle particitation, il s'efface derrière un hyperénonciateur qui fait autorité dans la collectivité concernée. Mais l'autorité appelle facilement le détournement. Ainsi dans l'énoncé suivant :

ô rage, ô désespoir, ô Pécresse ennemie

On a ici affaire à un détournement du vers célèbre du Cid. Il ne s'agit pas d'une subversion qui viserait à disqualifier Corneille mais d'une captation ludique qui place le locuteur à la place occupée par Don Diègue. En principe, le détournement est d'autant 
plus réussi que la modification est minimale; c'est le cas ici car où «vieillesse »a été remplacé par « Pécresse", ce qui permet de produire un énoncé dont la différence phonique avec l'énoncé source est minime. Mais on ne peut pas s'arrêter là : le fait qu'il s'agisse d'une aphorisation canonique (par sa forme, par son auteur et par sa notoriété) du Thésaurus de la littérature classique permet une fois de plus au locuteur de montrer un ethos de lettré, dont la cible, V. Pécresse, est censée dépourvue, elle qui précisément est « ennemie » de la culture qui rend possible cette énonciation même.

Le mécanisme est quelque peu différent dans cet autre détournement du Thésaurus littéraire qui utilise lui aussi le marquage rhétorique $\hat{O}+\mathrm{GN}$ :

O préparateurs sibyllins ! ô supputateurs inféconds ! ô ministres intègres !

Une telle aphorisation s'appuie sur l'actualité. Le 12 février 2009, le ministre de l'Éducation nationale de l'époque, en réponse à la demande des présidents des universités de repousser la mise en place de nouveaux concours, avait répondu :

Et aujourd'hui, un professeur sur deux qui est recruté par moi, n'est déjà pas passé par des systèmes de formation des maîtres. Il a tout simplement une licence ou une maîtrise, et il se présente à nos concours et il les a. Donc moi je n'ai pas absolument besoin d'entrer dans des discussions sibyllines avec les préparateurs à mes concours. Je suis recruteur. Je définis les concours dont j'ai besoin. Je garantis la formation professionnelle des personnels que je recruterai. Après, chacun nous suit, ou pas. ${ }^{7}$

Le jeudi 19 mars 2009, dans un article du Monde, X. Darcos s'était prononcé encore une fois sur le refus des universitaires d'accepter les réformes du gouvernement : "L'affaire ne se clive pas entre les bons (la gauche) et les méchants (la droite). Cette supputation est inféconde. Elle donne un exemple désastreux de notre jeunesse en privilégiant la querelle ou le préjugé plutôt que la controverse argumentée.» On voit que Darcos n'a jamais utilisé les groupes nominaux "préparateurs sibyllins", ni "supputateurs inféconds». C'est le créateur de l'énoncé manifestant qui fabrique une aphorisation dont le locuteur occupe la place de Ruy Blas, héros roturier qui dénonce la corruption de ministres issus de l'aristocratie. Il est clair qu'une telle captation ne fait sens que pour un destinataire 'humaniste'.

Les deux premiers groupes nominaux de l'énoncé utilisent, sur le mode de l'allusion, des mots employés par la cible, en l'occurrence X. Darcos, pour soutenir un certain point de vue. En revanche, le troisième groupe nominal, repris de V. Hugo, est automatiquement attribué à un énonciateur adversaire du ministre, provoquant une réinterprétation du début de l'énoncé qui inverse le point de vue ${ }^{8}$ : l'énonciateur de l'aphorisation n'est plus Darcos mais les manifestants. Mais on peut proposer une autre interprétation, selon laquelle les deux premiers groupes nominaux ont le ministre pour énonciateur et le troisième les manifestants, qui disqualifient indirectement le point de vue précédent en disqualifiant son énonciateur : ce dernier n'est pas «intègre ». Le point de vue du ministre dans les deux premiers groupes nominaux serait énoncé sur le mode de la subversion parodique. Comme dans toute parodie, l'énonciation s'accompagne d'indices de mise à distance qui permettent au destinataire de percevoir une dissonance ; à la différence de ce qui se passe dans l'ironie, le locuteur ridiculisé est censé identifiable. Ce qui suppose une forte connivence: le destinataire doit être suffisamment familier du discours subverti. Quant au point de vue des manifestants, il ne peut être pris en charge par l'énonciateur auquel s'identifierait le locuteur manifestant : en effet, l'énonciation de Ruy Blas est elle-même ironique, puisqu'il entend railler les ministres malhonnêtes. Comme le souligne Berrendonner, 
«faire de l'ironie, ce n'est pas s'inscrire en faux de manière mimétique contre l'acte de parole antérieur ou virtuel, en tout cas extérieur, d'un autre. C'est s'inscrire en faux contre sa propre énonciation, tout en l'accomplissant. » (1981:215)

Dans des exemples de ce type, on saisit à quel point les plans énonciatifs sont intriqués. Le détournement est une pratique de masque. Si, à sa manière, il dit 'je est un autre', c'est pour mieux s'investir des pouvoirs de cet autre ou le ruiner. On ne peut se contenter d'analyser de manière plus ou moins détaillée ces «jeux » : il est clair qu'ils sont indissociables précisément de ce discours manifestant et des acteurs qu'il implique. Derrière les « jeux » on entrevoit le sérieux d'affrontements politiques. Jouer ainsi avec la langue et les réminiscences culturelles, c'est imposer une certaine schématisation de la situation, légitimer l'espace à partir duquel on énonce.

\section{Conclusion}

De cette analyse, nous aimerions faire ressortir deux idées, pistes de réflexion pour des recherches ultérieures. Le discours manifestant que nous avons étudié est hétérogène ; il mêle un discours d'appareil (avec l'utilisation des slogans prototypiques, de formules qui s'inscrivent dans une culture de la contestation de gauche et qui implique une forte présence d'institutions et d'organismes tels que CNRS) et un discours 'humaniste' qui implique une culture lettrée, la connaissance des langues classiques, des langues étrangères et une dextérité verbale ostentatoire. Cette divergence recoupe en outre pour une bonne part l'opposition entre les énoncés sur les banderoles et les énoncés sur les affichettes. On peut la mettre en relation avec la présence massive des enseignants de lettres dans ces manifestations. Ce pourrait être d'ailleurs un des facteurs qui expliquent l'échec relatif de ces manifestations. L'identité énonciative que revendiquaient ces acteurs 'humanistes' ne pouvait être comprise, dans la majorité des cas, que par leurs pairs ; elle excluait même la majorité des universitaires. - Par ailleurs l'analyse de notre corpus montre que la notion de slogan politique est beaucoup trop liée à la vision traditionnelle du slogan comme énonciation collective, souvent fortement soumise, d'un point de vue linguistique, à la fonction poétique jakobsonienne. La notion plus compréhensive d'«énoncé manifestant» s'avère nécessaire. Au-delà du cas de ces manifestations universitaires de 2009, on peut se demander si la prolifération d'énoncés qui ne se montrent pas comme des slogans ne témoigne pas d'une évolution du discours manifestant contemporain, qui apparaît de plus en plus comme un lieu de tension entre les individualités et les identités collectives, associées à des partis, des syndicats ou d'autres institutions. Les manifestants ne se contentent plus de s'associer à des énoncés collectifs, ils produisent des énoncés qui se veulent singuliers, aux deux sens du mot : uniques (propres à un individu) et inattendus. Cela ne signifie pas nécessairement qu'il y ait à plus ou moins long terme substitution d'un type d'énoncé manifestant à l'autre; il peut s'agir d'une coexistence durable. Cela ne signifie pas non plus nécessairement que les énoncés qui se veulent singuliers soient idéologiquement hétérogènes; on a vu que ce n'était pas le cas dans notre corpus, où l'on constate surtout des variations sur une topique très réduite et très stable. Pour savoir si cette association entre les deux types d'énoncé manifestants est une manifestation d'une tendance de fond, il faudrait effectuer des comparaisons sur toute une période. 


\section{BIBLIOGRAPHIE}

Adam, Jean-Michel \& Marc Bonhomme. 1997. L'Argumentation publicitaire. Rhétorique de l'éloge et de la persuasion (Paris : Nathan Université)

Amossy, Ruth. 2010. La présentation de soi. Ethos et identité verbale (Paris : PUF)

Berrendonner, Alain. 1981. Éléments de pragmatique linguistique (Paris : Minuit)

Bres, Jacques \& Bernard Vérine. 2002. « Le bruissement des voix dans le discours : dialogisme et discours rapporté ", Faits de langues 19, 159-169

Bres, Jacques \& Alexandra Nowakowska. 2005. « Dis-moi avec qui tu "dialogues” ", je te dirai qui tu es... », Marges Linguistiques 9, 137-153

Delbecque, Nicole. 1990. «El lenguaje de la publicidad y su poder de sugestión. Anuncios publicitarios en el País semanal : los tópicos más frequentes y su formulación », Lingüistica española actual, XII, 2,197-213

Denton, Robert E. 1980. « The Rhetorical Functions of Slogans : Classifications and Characteristics ", Communication Quarterly $28: 2,10-18$

Ducrot, Oswald. 1984. Le Dire et le dit (Paris : Minuit)

Favre, Pierre (éd.) 1990. La Manifestation (Paris : Presses de la Fondation nationale des sciences politiques)

Garric, Nathalie. 1996. Etude des structures linguistico-discursives du slogan. (Paris : Thèse en linguistique, soutenue à l'Université Paris III)

Grinshpun, Yana. (à paraître) 2013. « Discours rapporté et “discours relais" : le cas du président et de la princesse », Cahiers de Praxématique

Grinshpun, Yana. 2012. « Le locuteur-marionnette. Entre mazarinades et sarkoziades », Grinshpun, Yana \& Judith Doggen (éds). Regards croisés sur la langue française : usages, pratiques, histoire (Paris : PSN)

Grunig, Blanche-Noël. 1990. Les mots de la publicité. L'architecture du slogan (Paris : Presses du CNRS)

Herrero Cecilia, Juan. 1995. « El eslogan publicitario en la prensa semanal y captación de las propiedades persuasivas de orasparemias », Paremia 4, 169-178

Herrero Cecilia, Juan. 1997. «El eslogan publicitario y los efectos comunicativos del juego de la ambigÜedad semántica », Paremia 6,337-341

Maingueneau, Dominique. 1987. Nouvelles tendances en analyse du discours (Paris, Hachette)

Maingueneau, Dominique. 1998. Analyser les textes de communication (Paris : Dunod)

Maingueneau, Dominique. 2004. « Hyperénonciateur et particitation », Langages 156, 111-127

Maingueneau, Dominique. 2012. Les phrases sans texte (Paris : Armand Colin)

Paveau, Marie-Anne. 2009. "Une énonciation sans communication : les tatouages scripturaux ", Paveau, Marie-Anne \& Pierre Zoberman (éds), Itinéraires ltc, (Paris : Cenel-L'Harmattan), 81-105

Paveau, Marie-Anne \& Pierre Zoberman (éds). 2009. « Corpographèses », numéro spécial de la revue Itinéraires ltc, (Paris : Cenel-L'Harmattan) 
Reboul, Olivier. 1975. Le slogan (Paris : PUF)

\section{NOTES}

1. Voir Carrier $1989 ; 1991$, et Jouhaud, 1951.

2. Ce corpus est donc limité. Les manifestations universitaires de 2009 se sont déroulées dans toutes les grandes villes françaises à forte population universitaire.

3. L'ironie du sort veut que X. Darcos soit agrégé de lettres et titulaire d'un doctorat d'études latines.

4. D. Maingueneau (1987: 33) appelle «anti-ethos » l'ethos opposé à celui de l'énonciateur. Dans les textes polémiques cet anti-ethos est souvent explicité. A travers les énoncés manifestants, qui mettent en scène l'ethos 'humaniste' des manifestants, se construit l'image d'un pouvoir au service de l'argent, inculte et ennemi du savoir.

5. Sur « l'affaire de la Princesse de Clèves et N. Sarkozy » voir Grinshpun 2013 (à paraître) et Grinshpun (2012).

6. (http://www.dailymotion.com/video/x8br0o_universites-pecresse-envoie-des-pre_news)

7. (http://www.rmc.fr/editorial/70932/darcos-aucune-raison-de-repousser-la-reforme/)

8. Ici «énonciateur» et "point de vue » sont pris au sens qu'ils ont dans la théorie de la polyphonie de Ducrot (1984)

\section{RÉSUMÉS}

Au cours du deuxième semestre 2009 l'université française connaît une grève quasi générale accompagnée par une série de manifestations. La manifestation est une situation de communication qu'on peut appréhender à la fois comme construction d'une communauté de conviction transitoire et comme espace de production et de "monstration » d'énoncés oraux et écrits. Dix manifestations contre les réformes de l'université et de la formation des maîtres, organisées dans la région parisienne, ont mis en scène ce que nous appelons le «discours universitaire manifestant ». Nous abordons ici ce discours comme une pratique inscrite dans une certaine conjoncture socio-politique. Ce travail se situe donc dans une perspective d'analyse du discours et vise à articuler fonctionnements linguistiques et situations de communication à travers les dispositifs d'énonciation.

During the second semester of the year 2009, the French academic community went on an almost universal strike. Ten street demonstrations against the governmental reforms concerning academic research and the training of future school teachers - took place. We consider street demonstration as a particular situation of communication that can be perceived as a "place" of production and an exposition of oral and written enunciations. In this article, we analyze these enunciations as a type of discourse that emerged in a particular socio-political context. Thus we adapt the discourse analysis perspective in order to articulate linguistic functions and communication situations through different modi of enunciation. 
INDEX

Mots-clés : communauté discursive, énoncé manifestant, hyperénonciateur, particitation, slogan

Keywords : demonstration, discourse community, enunciation, hyperenunciator, slogan, "particitation"

\section{AUTEUR}

YANA GRINSHPUN

Université Paris III, SYLED-Clesthia 